Voix et Images

voixetimages

\title{
Voix de femmes des années 1930
}

\section{Lori Saint-Martin}

Volume 39, numéro 2 (116), hiver 2014

Voix de femmes des années 1930

URI : https://id.erudit.org/iderudit/1025185ar

DOI : https://doi.org/10.7202/1025185ar

Aller au sommaire du numéro

Éditeur(s)

Université du Québec à Montréal

\section{ISSN}

0318-9201 (imprimé)

1705-933X (numérique)

Découvrir la revue

\section{Citer ce document}

Saint-Martin, L. (2014). Voix de femmes des années 1930. Voix et Images, 39(2),

9-15. https://doi.org/10.7202/1025185ar d'utilisation que vous pouvez consulter en ligne.

https://apropos.erudit.org/fr/usagers/politique-dutilisation/ 


\title{
VOIX DE FEMMES DES ANNÉES 1930
}

\author{
$++$ \\ LORI SAINT-MARTIN \\ Université du Québec à Montréal
}

Idée reçue tenace, à propos de la littérature québécoise : entre Angéline de Montbrun (1881-1882 en feuilleton) et le doublé Bonheur d'occasion-Le Survenant en 1945, ce serait le calme plat, voire le trou noir, du côté de l'écriture des femmes. Qui, aujourd'hui, en dehors d'une petite poignée de spécialistes, peut nommer des femmes ayant publié entre ces deux moments? Pourtant, les années 1920 en poésie et les années 1930 pour le roman ont vu émerger de nombreuses nouvelles voix de femmes qui, après une première réception parfois enthousiaste, parfois assassine, ont vite sombré dans le silence et l'oubli. C'est du désir de révéler un pan peu connu de notre histoire littéraire et de faire résonner ces voix de femmes qu'est né le présent dossier de Voix et Images.

Au-delà des angoisses et des incertitudes de tout aspirant écrivain devant la page blanche s'ajoutent, pour les femmes, des inquiétudes particulières. Vivre pour soi - pour s'exprimer, mener une carrière ou se faire un nom - ou pour se dévouer aux autres (son mari, ses enfants)? À cette époque, pour les femmes d'ici, seule la seconde option est socialement recevable. En 1919, monsieur Louis-Adolphe Paquet qualifie le féminisme de «mouvement pervers», d' " ambition fallacieuse [qui] entraîne hors de sa voie la plus élégante moitié de notre espèce, et menace les bases mêmes de la famille et de la société ${ }^{1}$ ». Une quinzaine d'années plus tard, en 1933, rien n'a changé au pays de Québec, puisque l'écrivain, éditeur et critique Albert Pelletier s'adresse ainsi à la femme de lettres Michelle Le Normand : "Chère madame, ne tenez donc pas à mon estime "comme écrivain" : je vous assure que je vous estime cent fois plus dans l'intimité de vos lettres et mille fois plus dans votre besogne quotidienne de vraie femme ${ }^{2}$.» Entre flatteries et subtil rappel à l'ordre (quelle étrange façon de s'adresser à une romancière !) se dessine une hiérarchie bien calculée : l'espace public («comme écrivain») convient cent fois moins à une femme que le cercle restreint de

1 Louis-Adolphe Paquet, «Le féminisme», Études et appréciations, t. IV : Nouveaux mélanges canadiens, Québec, Imprimerie franciscaine missionnaire, 1919, p. 3. 2 Cité dans Michel Lacroix, " "Mon petit commerce". Michelle Le Normand, femme de lettres et femme d'affaires», Chantal Savoie (dir.), Histoire littéraire des femmes. Cas et enjeux, Québec, Nota bene, coll. «Séminaires», 2010, p. 185. 
ses proches («l'intimité de vos lettres») et mille fois moins que son foyer (la «besogne quotidienne »). La femme artiste serait donc une «fausse femme» égarée par une chimère, une créature à la fois pitoyable et suspecte. Se crée ainsi, entre les aspirations personnelles et les attentes sociales, une tension vive, douloureuse, qui fragilise l'identité tant publique qu'intime et fait de la créatrice un être profondément divisé. Bon nombre de textes étudiés dans le cadre du présent dossier témoignent de ces déchirements et de ces tensions, dont on mesure mal, aujourd'hui, les effets délétères sur le sentiment de légitimité, la disponibilité mentale et l'énergie créatrice.

Mais il serait malhonnête de laisser entendre que les écrits des femmes n'ont rencontré que mépris et opprobre. Au contraire, la littérature d'ici était trop jeune durant les années qui nous intéressent, et son existence trop précaire, pour qu'on puisse se permettre de bouder les talents de la moitié de l'espèce, fût-elle ou non «la plus élégante». Un encart promotionnel pour Comme l'oiseau, recueil de poèmes de Jovette-Alice Bernier paru en 1926, reproduit un concert d'éloges de plumes tant masculines que féminines. Certes, on y trouve des remarques un brin condescendantes comme celles de Victor Barbeau («[c]hez les femmes, j’en connais peu de plus richement douées») ou d'Olivar Asselin («un talent naturel très remarquable chez une Canadienne»), mais Louis Francœur l'inclut au "nombre des vrais poètes», le $\mathrm{D}^{\mathrm{r}}$ Choquette dans La Presse la déclare «grand poète», Maurice Hébert recommande vivement cette lecture «pour apprendre de nouveau comme un poète sait dire des choses ailées» et La Revue populaire y voit «les meilleurs vers de l'année, dignes incontestablement d'un prix David ${ }^{3} »$. Alfred Desrochers a été le champion et le mentor de plusieurs écrivaines ${ }^{4}$; Louis Dantin parle, commentant leur poésie, d'un «élan féminin, presque féministe» qui «nous promet des richesses neuves ${ }^{5}$ »; les femmes récoltent alors de nombreux prix littéraires, fondent des revues et multiplient les pratiques littéraires ${ }^{6}$, investissent tant les "petits genres» moins valorisés, plus éphémères et souvent moins personnels - poésie champêtre, roman sentimental, billet, conte, légende, livre pour enfants - que les formes prestigieuses (roman, poésie) qui permettent l'élaboration d'une perspective et d'une esthétique propres. Si elles demeurent minoritaires - entre 1915 et 1960, elles forment, bon an mal an, entre

$$
++
$$

3 Les citations de ce paragraphe viennent de l'encart, conservé dans le fonds Albert-Ferland, MSS4, S5, SS2, D4, Montréal, Bibliothèque et Archives nationales du Québec. Le prix David couronnait alors un livre et non l'ensemble de l'œuvre d'un écrivain. 4 Voir Annette Hayward, «Paragraphes d'Alfred DesRochers, ou modernité et littérature féminine en 1931 », Solitude rompue, textes réunis par Cécile Cloutier-Wojciechowska et Réjean Robidoux, Ottawa, Éditions de l'Université d'Ottawa, coll. «Cahiers du Centre de recherche en civilisation canadienne-française», 1986, p. 156-165; et Marie-Claude Brosseau, Trois écrivaines de l'entre-deux-guerres : Alice Lemieux, Éva Senécal et Simone Routier, Québec, Nota bene, coll. «Études», 1998, 125 p. 5 Louis Dantin, "Après un siècle (1929-1929) », Gloses critiques, Montréal, Éditions Albert Lévesque, coll. «Les jugements », 1931, p. 165. Voir aussi Georges Bellerive, Brèves apologies de nos auteurs féminins, Québec, Librairie Garneau, 1920, 137 p., et Albert Pelletier, «Poèmes de jeunes filles », Carquois, Montréal, Librairie d'Action canadienne-française, coll. «Les jugements", 1931, p. 102-134. 6 Pour les informations contenues dans le reste du paragraphe, se rapporter à l'article fondateur de Lucie Robert, «D'Angéline de Montbrun à La chair décevante. La naissance d'une parole féminine autonome dans la littérature québécoise», Études littéraires, vol. XX, n 1, printemps-été 1987, p. 99110, repris dans Lori Saint-Martin (dir.), L'autre lecture. La critique au féminin et les textes québécois, t. I, Montréal, XYZ éditeur, coll. «Documents», 1992, p. 41-50. 
quinze et vingt pour cent de la population écrivante ${ }^{7}-$, leur succès, voire leur triomphe, semble assuré.

Et puis tout s'effrite, tout s'envole. Presque toutes ces femmes sont aujourd'hui oubliées, leurs livres épuisés et rarement commentés, leur présence effacée, leur reconnaissance presque nulle ${ }^{8}$.

Grâce aux travaux déjà menés, nous connaissons à peu près les raisons et les circonstances de l'essor de l'écriture des femmes - dans le journalisme ${ }^{9}$, puis le théâtre $^{10}$ au tournant du Xx ${ }^{\mathrm{e}}$ siècle ; dans la poésie ${ }^{11}$, surtout à partir des années 1920 ; et dans le roman ${ }^{12}$ au cours de la décennie suivante. Cette période est décisive pour le statut social des femmes et, de façon concomitante, pour leur fortune littéraire ${ }^{13}$. En effet, du côté social, elles obtiennent le droit de vote au fédéral et se regroupent pour le revendiquer au provincial, arrachent certains gains à un gouvernement hésitant dans la foulée de la commission Dorion, tenue en 1929, et accèdent à partir de 1908 au cours classique ${ }^{14}$. Pour ce qui est de leur fortune littéraire, certaines «héritières » - pour la plupart des femmes issues de familles d'écrivains, dotées d'un certain capital financier et culturel - profitent de nouvelles tendances comme l'industrialisation des journaux, qui a entraîné la création de pages féminines, ou encore l'émergence de débouchés comme le roman d'amour, la littérature jeunesse ou les romans-feuilletons de la radio ${ }^{15}$. C'est aussi à partir de ce moment qu'on les voit parfois se regrouper, comme le feront des femmes poètes à Québec et d'autres, autour d'Alfred DesRochers, à Sherbrooke, tandis que des éditeurs comme Albert Lévesque, créateur de la collection «Les romans de la jeune génération", où paraîtront La chair décevante et Dans les ombres, leur ouvrent leurs portes.

$$
+++
$$

7 Lucie Robert, «D'Angéline de Montbrun à La chair décevante», p. 42.8 Pour une étude approfondie de la réception de La chair décevante et de Dans les ombres, voir Daniel Chartier, L'émergence des classiques. La réception de la littérature québécoise des années 1930, Montréal, Fides, coll. «Nouvelles études québécoises», 2000, p. 177200. 9 Voir Aurélien Boivin et Kenneth Landry, «Françoise et Madeline, pionnières du journalisme féminin au Québec», Voix et Images, vol. IV, n 2, décembre 1978, p. 233-243; et Mary Jean Green, «The "Literary Feminists" and the Fight for Women's Writing in Québec ", Journal of Canadian Studies/Revue d'études canadiennes, vol. XX, $\mathrm{n}^{\circ}$ 1, 1986, p. 128-143. Voir aussi le roman de Germaine Guèvremont, Tu seras journaliste, paru en feuilleton dans Paysana en 1939-1940 (Tu seras journaliste et autres œuvres sur le journalisme, édition critique établie par David Décarie et Lori Saint-Martin, Montréal, Presses de l'Université de Montréal, coll. «Les écrits de Germaine Guèvremont. Euvres de fiction", 2013, 244 p.). 10 À divers titres, Madeleine (Anne-Marie Gleason) et Éva Circé-Côté figurent parmi les premières femmes représentées sur une scène professionnelle, en 1902 pour la première (en lever de rideau) et 1903 pour la seconde (en affiche principale). Voir Lucie Robert, Apprivoiser la modernité théâtrale. La pièce en un acte de la Belle Époque à la Crise. Anthologie, Québec, Nota bene, 2012, 263 p., et "Les stratégies éditoriales des premières femmes auteures dramatiques", Chantal Savoie (dir.), Histoire littéraire des femmes, p. 105-166. 11 Voir Lucie Robert et Corine Bola, "La poésie "féminine" de 1929-1940: une nouvelle approche», Atlantis, vol. IV, n 1 , automne 1978, p. 55-62, et Raija H. Koski, «La poésie féminine des années vingt: Jovette Bernier», Lori Saint-Martin (dir.), L'autre lecture, p. 51-56. 12 Voir Christl Verduyn, «La prose féminine québécoise des années 1930 », Québec Studies, n 8, printemps 1988, p. 43-57, repris dans Lori Saint-Martin (dir.), L'autre lecture, p. 57-72. 13 Voir Lucie Robert, «D’Angéline de Montbrun à La chair décevante»; Isabelle Boisclair, Ouvrir la voie/x. Le processus constitutif d'un sous-champ littéraire féministe au Québec (1960-1990), Québec, Nota bene, coll. «Littérature(s)», 2004, p. 71-111; Denis Saint-Jacques et Lucie Robert (dir.), La vie littéraire au Québec, t. VI: 1919-1933. Le nationaliste, l'individualiste et le marchand, Québec, Presses de l'Université Laval, 2010, p. 2. 14 Collectif Clio, L'histoire des femmes au Québec depuis quatre siècles, Montréal, Le Jour, 1992, p. 339.15 Voir Lucie Robert, «D'Angéline de Montbrun à La chair décevante»; et Isabelle Boisclair, Ouvrir la voie/x. 
On peut donc parler d'un important essor de l'écriture des femmes : avant 1938, elles publient deux cent trente-huit ouvrages ${ }^{16}$. Inventoriant la production féminine, Isabelle Boisclair distingue une "période balbutiante » entre 1900 et 1914, suivie d'une époque de progression constante, de 1915 à 1930, puis d'une décennie stable, de 1931 à $1942{ }^{17}$. Mais les chiffres ne disent pas tout. Il faut savoir qui étaient ces femmes, quels genres elles ont investis, quelles stratégies elles ont pratiquées et quel accueil elles ont reçu. À ce titre, les travaux de Lucie Robert, d'Isabelle Boisclair et de Daniel Chartier déjà mentionnés, ainsi que, plus récemment, l'important chantier ouvert par Chantal Savoie autour de l'histoire littéraire des femmes au tournant du Xxe siècle ${ }^{18}$, sont d'un précieux secours. Beaucoup plus rares jusqu'à présent, toutefois, ont été les études portant sur les œuvres elles-mêmes ${ }^{19}$ plutôt que sur les conditions de leur parution, les stratégies professionnelles ou le contexte socioculturel. C'est dans cette voie encore peu explorée que devrait s'engager au moins une partie du travail critique à venir.

Mais comment étudier aujourd'hui ces textes, dont la valeur littéraire est loin d'être toujours insigne ${ }^{20}$ ? Pour commencer, il faudrait les situer dans le contexte littéraire "général» de leur époque et situer leurs pratiques génériques, leurs stratégies discursives, les sujets traités et le ton adopté par rapport à ceux employés par les hommes de la même période. Il faudra donc mener des études comparatives hommesfemmes, ce qu'aucun critique n'a encore fait.

Une question centrale demeure : celle, déjà esquissée par Lucie Robert, des tensions et des paradoxes que soulève la pratique de divers genres populaires. Comment les femmes ont-elles navigué entre les contraintes et les occasions nouvelles? L'ouverture d'espaces propres - la "page féminine» des grands quotidiens et des magazines, par exemple - a-t-elle favorisé l'apparition de discours nouveaux ou la simple remise en circulation d'idées reçues? Par ailleurs, puisque les auteurs de cette époque ont eu tendance à investir plus d'un genre, ajoutant à la pratique du journalisme celle de la poésie, du roman ou du théâtre, il faudrait mener une lecture transversale de certains trajets, liant à l'étude des œuvres des réflexions sur les stratégies

$$
++
$$

16 Lucie Robert, «D’Angéline de Montbrun à La chair décevante», p. 42.17 Isabelle Boisclair, Ouvrir la voie/x, p. 81. 18 Voir Histoire littéraire des femmes, le dossier «Sans livres mais pas sans lettres. Renouveler l'histoire des pratiques d'écriture des femmes», qu'elle a dirigé avec Marie-José des Rivières (Recherches féministes, vol. XXIV, nº 1, 2011, p. 1-226), ainsi que de nombreux articles, dont «La page féminine des grands quotidiens montréalais comme lieu de sociabilité littéraire au tournant du xxe siècle», Tangence, nº 80, hiver 2006, p. 125-142; et "Des salons aux annales: les réseaux et associations des femmes de lettres à Montréal au tournant du Xx siècle», Voix et Images, vol. XXVII, n² 2, hiver 2002, p. 238-253. 19 Voir néanmoins Raija H. Koski, «La poésie féminine des années vingt: Jovette Bernier»; Adrien Rannaud, « Du silence au cri. La parole féminine solitaire dans La chair décevante", Studies in Canadian Literature/Études en littérature canadienne, vol. XXXVII, n 1, 2012, p. 141-152; Lucie Robert, "Yvette Mercier-Gouin ou le désir du théâtre ", L'Annuaire thêâtral, n 46, 2009, p. 117-137; Lucie Robert et Corine Bola, «La poésie "féminine" de 1929-1940: une nouvelle approche»; Lori Saint-Martin, «La chair décevante de Jovette Bernier: le Nom de la Mère", Tangence, n 47, 1995, p. 112-124; Christl Verduyn, "Une voix féminine précoce au théâtre québécois: Cocktail (1935), d'Yvette Ollivier Mercier-Gouin », Theatre Research in Canada/Recherches thêâtrales au Canada, vol. XI, nº 1, printemps 1990, p. 48-58, repris dans Lori Saint-Martin (dir.), L'autre lecture, p. 73-84, et certains textes de Chantal Savoir (dir.), d'Histoire littéraire des femmes. $\quad 20$ Les réflexions qui suivent ont surtout rapport à la prose narrative, mais la poésie et le théâtre devraient être soumis au même genre d'examen critique. 
d'autopromotion ${ }^{21}$. Un retour sur l'ensemble des textes critiques consacrés à l'écriture des femmes d'alors, de la réception critique immédiate aux essais et aux synthèses, s'impose également.

Une autre question vaste et complexe mérite l'attention: celle de la conformité aux discours existants ou de la rupture par rapport à ceux-ci. Une bonne partie de la production des femmes de l'époque (et on pourrait évidemment en dire autant de celle des hommes) est de nature édifiante ou du moins ne remet en cause aucune idée reçue. Mais la question est-elle aussi simple? La présence d'éléments traditionnels (prégnance du religieux, éloge de la vie campagnarde, valorisation du mariage et de la maternité) voue-t-elle d'emblée les œuvres féminines à la médiocrité ? Ne faudraitil pas s'interroger sur la possible valeur stratégique de certains discours, se demander par exemple, avec Mary Jean Green, si, comme les féministes d'autres pays, les Québécoises ne s'abritaient pas derrière des discours en apparence conformes non seulement pour entrer dans le champ littéraire, mais aussi pour y faire entendre en sourdine un propos distinct 22 ? La même figure n'a pas toujours le même sens : pour donner un exemple typique de l'époque, on peut invoquer Dieu pour se soumettre, pour le prendre à partie ou encore pour l'érotiser, comme on le fera couramment avec la figure christique. De même, si le thème de l'amour risque de confiner la femme dans la dépendance domestique, il ouvre aussi la porte à un discours de la sensualité ou encore de la révolte (contre les hommes négligents ou volages, contre les préjugés et tabous, etc.). Et en effet, les critiques et les revendications fusent: on fustige l'éducation réservée aux jeunes filles, la stigmatisation du corps et du désir ou encore les contraintes sociales imposées au sexe féminin ${ }^{23}$. Surgissent aussi des thèmes troublants comme les amours mixtes (personnes d'origine, de langue ou de religion différentes) ou hors mariage, la maternité vue de l'intérieur, les agressions sexuelles, le voyage comme signe d'autonomie, l'exploration de la ville, l'amitié entre femmes et bien d'autres.

Nombre de stratégies littéraires méritent aussi qu' on s'y attarde. Tout en cherchant la spécificité de chaque voix, on pourra cerner les traits communs, s'intéresser aux lieux représentés, interroger les choix formels (structures, voix narratives, tons) et débusquer les métaphores et les motifs récurrents. Par exemple, la coprésence dans le texte de deux amies à la personnalité contrastée permet d'opposer deux attitudes devant la vie, l'une plus soumise, l'autre plus rebelle; le voyage rime souvent avec le métissage identitaire et l'ouverture à l'altérité, etc.

Le dénouement des romans pose souvent un problème particulier. On pourrait se demander dans quelle mesure une fin qui prend la forme d'une punition ou d'un renoncement - la folie de Didi dans les dernières pages de La chair décevante, le rejet du projet d'aller vivre avec un homme divorcé à New York dans Moments de vertige

$$
++
$$

21 Voir Michel Lacroix, "“Mon petit commerce" »; et Michel Lacroix et Nadia Zurek, "Une journaliste francoaméricaine au seuil de l'avant-garde. L'espace des possibles d'Yvonne Le Maître (1876-1954)", Recherches féministes, vol. XXIV, n ${ }^{\circ}$ 1, 2011, p. 77-99. 22 Mary Jean Green, "The "Literary Feminists" and the Fight for Women's Writing in Québec». Cet article, pourtant essentiel, est peu cité par les critiques québécois. 23 Voir Christl Verduyn, «La prose féminine québécoise des années 1930 »; et Isabelle Boisclair, Ouvrir la voie/x. 
(1931) de Maxine (pseudonyme de Marie-Caroline-Alexandra Bouchette ${ }^{24}$ ), l'abandon du journalisme au profit du mariage et de la vie à la campagne dans Tu seras journaliste de Germaine Guèvremont - autorise et «fait passer» l'exploration, tout au long du roman, de parcours transgressifs, ainsi que l'expression d'une critique des normes sociales. De même, le mélodrame (la multiplication des accidents, des morts, des secrets dévoilés), s'il est souvent signe d'un manque de métier, permet parfois quelques audaces (l'expression d'une colère ou d'une révolte voilée, une réflexion sur la légitimité et donc sur la loi et la répression). Au-delà des maladresses certaines et du conformisme au moins apparent, y a-t-il ou non une volonté de renouveau formel et social dans ces textes? S'exprime-t-elle ouvertement ou de manière codée, secrète, inter-dite? Voilà sans doute la question centrale, à laquelle le présent dossier apporte déjà quelques éléments de réponse.

Les articles s'articulent en deux sous-ensembles complémentaires, le premier portant sur les genres non canoniques investis par les femmes, le second consacré aux stratégies textuelles qu'elles emploient dans les genres consacrés: roman et poésie.

Les trois premiers articles portent sur des genres parallèles ou mineurs, des écrits de nature plutôt éphémère et pratiqués de manière relativement conventionnelle, mais où émergent les balbutiements d'une subjectivité et d'une voix nouvelles. Ainsi, Manon Auger examine les rares œuvres diaristiques féminines publiées - le genre a longtemps été dominé par les hommes - et les motifs qui ont servi à justifier la prise de parole féminine: le désir de servir Dieu ou quelque bonne cause, par exemple. Affirmer un «je» féminin souverain, une vision propre, n'allait pas de soi. Dans la même veine, Patricia Smart étudie le bouleversant journal intime inédit de la romancière Michelle Le Normand, rédigé à partir des années 1920 - cet article déborde donc légèrement la période retenue -, qui révèle une tension permanente entre ambition propre, obligations conjugales et nécessité de se consacrer aux autres, en l'occurrence une famille et un mari lui-même romancier, Léo-Paul Desrosiers. Enfin, Chantal Savoie se penche sur d'autres genres mineurs, la chronique et le billet journalistiques, et retient deux types de pratiques, l'une plus traditionnelle, axée sur la vie rurale et le passé, l'autre plus moderne et urbaine. Les trois articles mettent en lumière, chacun à sa façon, les modalités d'une venue à l'écriture soumise à des contraintes génériques et morales, certes, et souvent marquée par la culpabilité et le besoin de se justifier, mais soustendue par une volonté réelle d'expression et de professionnalisation.

Au détour des formes intimes émergent parfois déjà un ton, un style, une quête artistique en somme. À plus forte raison, le roman et la poésie permettent un tel investissement et supposent un projet créateur davantage voulu et assumé. Le deuxième sous-ensemble du présent dossier a pour fil conducteur non seulement cet investissement à la fois esthétique et éthique, mais aussi le désir féminin qu'on commence alors à dire haut et fort. Daniel Chartier analyse l'érotisation de l'Amérindien dans Mon sauvage de Laure Berthiaume-Denault (1938) et les renversements que le texte opère en mettant en scène un regard féminin désirant posé sur un corps masculin

24 Denis Saint-Jacques et Lucie Robert (dir.), La vie littéraire au Québec, t. VI : 1919-1933. Le nationaliste, l'individualiste et le marchand, p. 390. 
doublement autre. Si certains stéréotypes demeurent, cet investissement libidinal et amoureux constitue un point fort de la production romanesque de l'époque. Véronique Lord se penche sur une autre héroïne non conforme, celle de Dans les ombres (1931), d'Éva Senécal, femme mariée qui vit une idylle amoureuse et sensuelle avec un autre homme, Américain de surcroît. Les théories féministes de l'agentivité permettent de suivre pas à pas le parcours d'une protagoniste qui, si elle finit par rentrer dans le rang, n'en est pas moins libre et transgressive. Enfin, Adrien Rannaud s'intéresse, dans deux œuvres de Jovette-Alice Bernier - le roman La chair décevante (1931) et le recueil de poésie Les masques déchirés (1932) -, à l'expression de la sensualité. Si la critique a signalé maintes fois sa présence, Adrien Rannaud montre en détail comment elle se manifeste, entre autres dans la réitération de mots comme «chair» ou «volupté», et dans l'accent mis sur les impressions fugaces, les plaisirs et les corps. Ainsi, les trois articles convergent pour faire des années 1930 une décennie où les créatrices disent leur désir, font l'éloge du corps et du plaisir, érotisent la figure de l'homme ethniquement Autre à une époque où le mariage mixte est condamné, rejettent les normes sociosexuelles, pratiquent une écriture sensuelle et proposent un regard, une voix autres.

De façon plus générale, donc, c'est du côté de l'ambivalence, des tensions, des impasses et des non-dits qu'on trouve une importante clé de lecture de l'ensemble de cette production. Entre les interdits et les désirs naissent les tensions, les apories, les paradoxes. L'écriture des femmes navigue entre tradition et innovation, rôles féminins imposés et recherche de nouvelles identités, ambition personnelle et obligations familiales, désir de légitimation et désir de rupture, reprise des modèles connus et quête éthique et artistique nouvelle. Le chantier est vaste, et la matière, abondante. Le présent dossier ouvre des pistes variées et prometteuses. 